\title{
Diagnosis of Levothyroxine Pseudo-malabsorption: The Results of Standardised Testing Protocol
}

\author{
Mehmet Sözen ${ }^{1}$, Zeynep Canturk ${ }^{1}$, Berrin Cetinarslan ${ }^{1}$, Ilhan Tarkun², Emre Gezer ${ }^{1}$ and Alev Selek ${ }^{1}$ \\ ${ }^{1}$ Department of Endocrinology and Metabolism, Faculty of Medicine, Kocaeli University, Kocaeli, Turkey \\ ${ }^{2}$ Department of Endocrinology and Metabolism, Anadolu Medical Center, Kocaeli, Turkey
}

\begin{abstract}
The levothyroxine absorption test for the assessment of pseudo-malabsorption in patients with refractory hypothyroidism has not been standardised. The aim of this observational study was to describe a protocol for levothyroxine (LT-4) absorption test in patients with refractory hypothyroidism, to report possible side effects and to emphasise the importance of pseudo-malabsorption in the differential diagnosis. The results of 10 patients, who underwent LT-4 absorption tests because thyroid stimulating hormone suppression could not be achieved despite the need for LT-4 $>3 \mathrm{mcg} / \mathrm{kg} / \mathrm{day}$, were retrospectively analysed. When compared with basal free T4 (fT4), a statistically significant increase in fT4 was observed after the first hour $(p=0.012)$. fT4 reached its peak level and plateau at the $4^{\text {th }}$ hour. The fT4 peak level increased 3.25 times compared to baseline. The shorter LT-4 absorption test with low doses may provide an alternative method to the commonly used longer protocols with higher doses to rule out malabsorption.
\end{abstract}

Key Words: Levothyroxine absorption test, Pseudo-malabsorption, Resistant hypothyroidism.

How to cite this article: Sözen M, Canturk Z, Cetinarslan B, Tarkun I, Gezer E, Selek A. Diagnosis of Levothyroxine Pseudo-malabsorption: The Results of Standardised Testing Protocol. J Coll Physicians Surg Pak 2021; 31(09):1099-1101.

\section{INTRODUCTION}

Hypothyroidism is a common endocrine disorder with an annual incidence of $1-2 \%$. In patients with hypothyroidism levothyroxine (LT-4) requirement is approximately $1.2-1.6 \mathrm{mcg} / \mathrm{Kg} /$ day in most patients. In some patients, clinical and laboratory hypothyroidism cannot be managed in spite of high doses. ${ }^{1}$ LT-4 malabsorption is a commonly known entity for some diseases. Common causes include gastrointestinal (GI), hepatic and pancreatic diseases, gastrointestinal surgical procedures, some drugs, dietary interactions, heart disease or pregnancy. However, the mostcommon cause is poor/non-compliance with oral LT-4 treatment. ${ }^{2} A n$ objective way for differentiating patient's non-compliance from malabsorption is LT-4 oral absorption testing. ${ }^{3}$ Unfortunately, there are no vast number of case studies in the literature, indicating standardization of the test.

The aim of this study was to describe a protocol for a faster LT-4 absorption test in patients with treatment-resistant hypothyroidism, to report possible side effects and to emphasise the importance of a possible diagnosis of pseudo-malabsorption.

Correspondence to: Mehmet Sözen, Department of Endocrinology and Metabolism, Kocaeli University, Kocaeli, Turkey

E-mail: mehmetsozen07@gmail.com

Received: January 29, 2021; Revised: May 01, 2021; Accepted: May 18, 2021

DOI: https://doi.org/10.29271/jcpsp.2021.09.1099

\section{METHODOLOGY}

In this study, the test results of patients, who underwent LT-4 absorption test at Endocrinology Clinic in the Kocaeli University Hospital, were evaluated retrospectively. The results between 05.09.2017-29.10.2019 were examined. The test was carried out after hospitalisation to monitor cardiac side effects and to make sure that the agent was administered. Ten patients were included in this study. The test was performed in the cases of overt hypothyroidism despite high dose LT-4 therapy of $>3 \mathrm{mcg} / \mathrm{Kg} /$ day. Before the test, Gl, liver and kidney diseases were excluded in all cases. Basal thyroid status, treatment doses, clinical and demographic findings were evaluated. After an overnight fasting, the patients were given about twice their usual LT-4 doses up to maximum 1 mg LT-4 (except for the pregnant patient). All drugs were given under supervision of nursing staff. Afterwards thyroid stimulating hormone (TSH) and free T4 (fT4) levels were measured at 0, 60, $120,180,240,300$ and 360 minutes. TSH and fT4 measurements were performed using electrochemiluminescence immunoassays. The patients were monitored in case of any cardiovascular side effects during and after the test. No complication related to LT-4 absorption test was observed in any patient in the following 24 hours.

All statistical analyses were performed using IBM SPSS for Windows version 20.0 (IBM Corp., Armonk, NY, USA). Shapiro-Wilk tests were used to test the normality of data distribution. Continuous variables were expressed as mean \pm standard deviation. Comparisons of normally distributed continuous paired variables between the times were performed using the paired sample t-test. Atwo-sided pvalue $<0.05$ was considered statistically significant. 
Table I: The Diagnosis, basal thyroid satus, LT-4 doses and test results of the patient.

\begin{tabular}{|c|c|c|c|c|c|c|c|}
\hline Patient & Diagnosis & LT-4 dose & Basal TSH & Basal FT4 & LT-4 test dose & Peak FT4 & Nadir TSH \\
\hline 1 & Hashimoto thyroiditis & $300(3.84 \mathrm{mcg} / \mathrm{kg})$ & 287 & $<0.25$ & 600 & 0.87 & 259 \\
\hline 2 & Papillary thyroid cancer (operated) & $350(4.21 \mathrm{mcg} / \mathrm{kg})$ & 308 & $<0.25$ & 700 & 1.69 & 283 \\
\hline 3 & Papillary thyroid cancer (operated) & $400(4.87 \mathrm{mcg} / \mathrm{kg})$ & 258 & 0.25 & 800 & 1.33 & 234 \\
\hline 4 & Hashimoto thyroiditis + pregnancy & $500(6.25 \mathrm{mcg} / \mathrm{kg})$ & 143 & $<0.25$ & 600 & 0.38 & 130 \\
\hline 5 & Papillary thyroid cancer (operated) & $800(9.1 \mathrm{mcg} / \mathrm{kg})$ & 88 & 0.6 & 900 & 2.14 & 53 \\
\hline 6* & Papillary thyroid cancer (operated) & $300(3.15 \mathrm{mcg} / \mathrm{kg})$ & 188 & 0.3 & 700 & 0.75 & 151 \\
\hline 7 & Papillary thyroid cancer (operated) & $300(7.1 \mathrm{mcg} / \mathrm{kg})$ & 66 & 0.55 & 900 & 1.45 & 62 \\
\hline $8 * *$ & Papillary thyroid cancer (operated) & $600(3.06 \mathrm{mcg} / \mathrm{kg})$ & 59 & 0.29 & 1000 & 1.59 & 48 \\
\hline $9 * *$ & Multinodular goiter (operated) & $450(6.42 \mathrm{mcg} / \mathrm{kg})$ & 10 & 0.55 & 900 & 1.69 & 8 \\
\hline 10 & Hashimoto thyroiditis & $350(4.11 \mathrm{mcg} / \mathrm{kg})$ & 31 & 0.57 & 700 & 1.58 & 24 \\
\hline
\end{tabular}

\section{RESULTS}

A total of 10 patients were included in the study. The mean age of the patients was $44.4 \pm 10.8$. Body mass index (BMI) of each patient was $>25 \mathrm{Kg} / \mathrm{m}^{2}$, and 7 patients were obese (BMI $>30 \mathrm{Kg} / \mathrm{m}^{2}$ ). The mean TSH level was $143.8 \pm 110.3$ $\mu \mathrm{IU} / \mathrm{mL}$ during pre-test evaluation. Mean daily dose of LT4 was $435 \pm 161.7 \mathrm{mcg}$. LT-4 absorption test was performed with a standard protocol in all patients. For the pregnant woman, the test dose could not be doubled due to the pregnancy and therefore, normal fT4 levels could not be obtained. Daily LT-4 doses and levothyroxine absorption test results are listed below (Table I).

There was a statistically significant difference between the basal level of fT4 and fT4 level in the first hour after levothyroxine administration ( $p=0.012)$. The mean fT4 levels reached to normal range in the first hour following ingestion of LT-4. The peak level was seen in the fourth hour, but then the plateau (Figure 1).

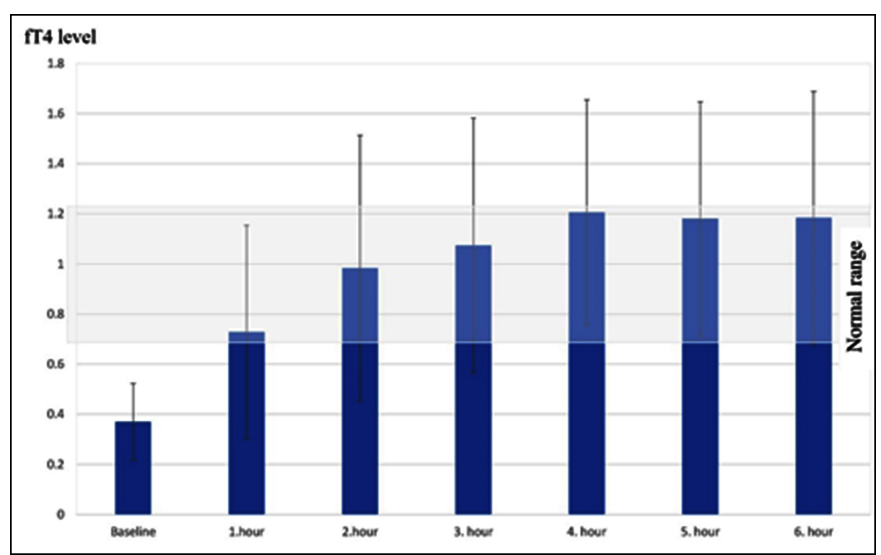

Figure 1: Six-hour levothyroxine absorption test results: fT4 levels after oral administration of levothyroxine.

\section{DISCUSSION}

The authors aimed to define a standard protocol for levothyroxine absorption testing in patients with refractory hypothyroidism to rule out levothyroxine malabsorption. The absence of a standard approach causes some diagnostic difficulties. In this study, successful results were achieved with lower LT-4 test doses in a shorter period refraining from unnecessary drug exposure.
The increased LT-4 requirement can be seen in many cases. Ain et al. first revealed the theory of pseudo-malabsorption in 1991 after evaluating four refractory hypothyroid cases despite high doses of oral thyroid hormone replacement therapy. ${ }^{4}$ The LT-4 absorption test is used to distinguish patients' non-compliance from malabsorption. ${ }^{3}$ Although various protocols have been described in the literature, there is no gold standard method for LT-4 absorption test. ${ }^{5}$ If the levothyroxine absorption rate calculated using tT4 with a non-isotope method is $\geq 60 \%$, it can be said that the absorption is normal. However, fT4 has largely supplanted tT4 in clinical practice. In studies, fT4 and tT4 showed high correlation even in patients with severe hypothyroidism. ${ }^{6}$ For this reason, fT4 has been suggested that it can be used instead of tT4 in LT-4 absorption test. ${ }^{6}$ The most preferred LT-4 dose is 1000 mcg as liquid or tablet form in the literature. ${ }^{3,5,6}$ However, we performed the 6 -hour test with lower doses of LT-4. The authors determined the LT-4 test dose of each patient as twofold of their daily doses but not exceeding $1000 \mathrm{mcg}$. A significant increase in plasma fT4 levels was achieved demonstrating the adequate absorption of LT-4.

In patients with hypothyroidism, fT4 usually increases one hour after LT-4 administration and peaks at 2 hours. ${ }^{5}$ In another study, fT4 reached its peak level in two hours and the mean increase was 4.7 times that of the average basal level. ${ }^{7}$ In this study, fT4 reached the normal reference range at the first hour and the peak level at the $4^{\text {th }}$ hour. The $\mathrm{ft} 4$ peak level increased 3.25 times compared to baseline.

In elderly patients with a history of cardiovascular disease, increased cardiac pressure load due to high thyroid hormone levels may lead to deterioration in cardiac functions. ${ }^{8}$ Successful responses to LT-4 absorption test was observed with lower doses of LT-4 than the doses reported in the literature. As a result, unnecessary dose was avoided, which is an important issue for the safety of the test, especially in patients with cardiovascular disease history. Both in the literature and in this study, there was no significant decrease in TSH levels in the 6-hour LT-4 absorption test. Therefore, we recommend fT4 measurements only in order to simplify the test and to be cost effective. 
Celiac disease may cause hypothyroid patients to require higher doses of LT-4 to maintain euthyroid status. ${ }^{8}$ In this study, the patients with celiac disease had a good compliance to their gluten-free diets and showed significant increase in fT4 levels during the LT-4 absorption test.

Three cases of thyroid cancer with LT-4 pseudomalabsorption have been reported in the literature and they were all female. Increased fT4 response to LT4 absorption test was observed in all three patients. ${ }^{9,10}$ Six of the10 patients in this study were previously diagnosed with thyroid cancer. All patients with thyroid cancer were female but, it was difficult to conclude a causal relationship with such a small number of patients. It can be promisingly predicted that frequent discontinuation to provide endogenous hypothyroidism for radioactive iodine treatment and wholebody radioiodine scan might cause intestinal edema.

The patients are still coming to outpatient clinic controls. Although the LT-4 dose was increased in two patients, normalisation of TSH could not be achieved. Therefore, intravenous LT-4 was added to the treatment of these patients and euthyroidism was achieved in their follow-up. All patients are currently being followed up stably.

\section{CONCLUSION}

Low-dose LT-4 absorption test can be used successfully to avoid the effects of high-dose levothyroxine, especially in elderly patients and patients with heart disease.

\section{ETHICAL APPROVAL:}

All procedures performed in this study involving human participants were in accordance with the 1964 Declaration of Helsinki and its later amendments or comparable ethical standards. The study was approved by the Ethics Committee of the University of Kocaeli with the project number KÜ GOKAEK 2019/124.

\section{PATIENTS' CONSENT:}

Informed consents were obtained from all individual participants included in the study.

\section{CONFLICT OF INTEREST:}

The authors declared no conflict of interest.

\section{AUTHORS' CONTRIBUTION:}

MS: Study concept and design, analysis and interpretation of data, writing of article, critical revision of the manuscript.

ZC: Analysis and interpretation of data, critical revision of the manuscript.

BC: Study concept and design, critical revision of the manuscript, study supervision.

IT: Writing of article, study supervision.

EG: Analysis and interpretation of data, writing of article.

AS: Study concept and design, writing of article, critical revision of the manuscript.

\section{REFERENCES}

1. Gaitonde DY, Rowley KD, Sweeney LB. Hypothyroidism: An update. Am Fam Physician 2012; 86(3):244-51.

2. Watts NB, Blevins LS. Endocrinology. JAMA 1994; 271(21): 1666-8.

3. Lips DJ, Van Reisen MT, Voigt V, Venekamp W. Diagnosis and treatment of levothyroxine pseudomalabsorption. Neth J Med 2004; 62(4):114-8.

4. Ain KB, Refetoff S, Fein HG, Weintraub BD. Pseudomalabsorption of levothyroxine. JAMA 1991; 266(15):2118-20.

5. Balla $M$, Jhingan RM, Rubin DJ. Rapid levothyroxine absorption testing: A case series of nonadherent patients. Int J Endocrinol Metab 2015; 13(4):e31051. doi: 10.5812/ ijem. 31051.

6. Sun GEC, Pantalone KM, Faiman C, Gupta M, Olansky L, Hatipoglu $B$. The clinical utility of free thyroxine in oral levothyroxine absorption testing. Endocr Pract 2014; 20(9):925-9. doi: 10.4158/EP13487.OR.

7. Soares RM, de Figueiredo RM, Melo Dantas MN, Solano Brito MV, Marinho MV, Pires Sousa AG, et al. Rapid levothyroxine (LT4) absorption test for diagnosis of LT4 pseudomalabsorption \& case report and proposal of a cutoff point. J Endocrinol Diabetes Obes 2016; 4(1):1083.

8. Rizzo LFL, Mana DL. Treatment of hypothyroidism in special situations. Med (BAires) 2020; 80(6):83-93.

9. Tönjes A, Karger S, Koch CA, Paschke R, Tannapfel A, Stumvoll $M$, et al. Impaired enteral levothyroxine absorption in hypothyroidism refractory to oral therapy after thyroid ablation for papillary thyroid cancer: Case report and kinetic studies. Thyroid 2006; 16(10):1047-51. doi: 10.1089/thy. 2006.16.1047.

10. Nagaoka T, Miyakoshi H, Takamura T, Nagai $Y$, Matsushita $\mathrm{S}$, Kaneko $\mathrm{S}$, et al. A case of refractory hypothyroidism requiring daily intravenous thyroxine. J Int Med Res 2002; 30(4):463-5. doi: 10.1177/147323000203000418. 